\title{
Accurate crab cavity modeling for the high luminosity Large Hadron Collider
}

\author{
D. R. Brett" and R. B. Appleby ${ }^{\dagger}$ \\ School of Physics and Astronomy, The University of Manchester, Manchester M13 9PL, United Kingdom \\ and Cockcroft Institute, Daresbury Laboratory, Warrington WA4 4AD, United Kingdom \\ R. De Maria, J. Barranco Garcia, ${ }^{\ddagger}$ and R. Tomás Garcia \\ CERN, Geneva 23, CH-1211, Switzerland \\ B. Hall and G. Burt \\ Lancaster University, Lancaster LA1 4YR, United Kingdom \\ and Cockcroft Institute, Daresbury Laboratory, Warrington WA4 4AD, United Kingdom
}

(Received 17 March 2014; published 2 October 2014)

\begin{abstract}
As part of the Large Hadron Collider high luminosity upgrade it is proposed to include crab cavities in the lattice in order to enhance the luminosity. For one proposed cavity design the dynamics of the cavity is considered in terms of its impact upon the dynamic aperture of the machine. Taylor maps of the cavity are created and used to perform this analysis with a full assessment of their validity. Furthermore from these Taylor maps, symplectic methods are developed further, guided by the knowledge gained in the study of the physics contained in them.
\end{abstract}

DOI: 10.1103/PhysRevSTAB.17.104001

PACS numbers: 41.85.Ja, 29.20.db, 41.75.-i

\section{INTRODUCTION}

The LHC is a two beam synchrotron proton collider located at CERN, Geneva. The machine is now operating successfully, delivering proton-proton luminosity to its several experiments, and the luminosity upgrade for the two high luminosity (HL) experiments ATLAS (located at interaction point 1, IP1) and CMS (located at IP5) is now being planned. The goal is a leveled luminosity of $5 \times 10^{34} \mathrm{~cm}^{-2} \mathrm{~s}^{-1}$, requiring a virtual peak luminosity of $2 \times 10^{35} \mathrm{~cm}^{-2} \mathrm{~s}^{-1}[1]$.

The LHC bunch spacing of $25 \mathrm{~ns}$ necessitates the use of a large beam crossing angle at the IP to avoid additional (parasitic) interactions between the bunches. This results in a reduction of the geometric overlap of bunches, and hence a reduction in luminosity. This rather inefficient way of colliding the proton bunches can be mitigated by tilting the bunches in the plane of the crossing angle, as shown in Fig. 1, using crab cavities, thus recovering a head-on collision geometry $[2,3]$.

The proposed cavity design considered in this paper is the four rod design (4RCAV) shown in Fig. 2. The cavity

*dave.brett@hep.manchester.ac.uk

robert.appleby@manchester.ac.uk

Present address: Ecole Polytechnique Fédérale de Lausanne (EPFL), CH-1015 Lausanne, Switzerland.

Published by the American Physical Society under the terms of the Creative Commons Attribution 3.0 License. Further distribution of this work must maintain attribution to the author $(s)$ and the published article's title, journal citation, and DOI. version studied was the 2011 design [4]. There are two other proposed cavity designs also under consideration which are also based around quarter wave resonators [5]. The 4RCAV is approximately $700 \mathrm{~mm}$ long with a continuous circular aperture of $84 \mathrm{~mm}$ through which the beam passes, as shown in Fig. 2(b). The cavity design is based upon four quarter wave resonators positioned colinearly about the beam axis, where the length of the resonators is the dominant factor determining the frequency of the cavity. In particular this constrains the cavity's compactness to be limited by its length. The cavity's electric field peaks on the tips of the rods with various modes formed by the polarities of the four rod tips. The cavity has three symmetry planes about the $x, y$, and $z$ axis as shown in the figure; this is important in terms of considering the cavity fields as it removes the possibility of skew components. The complete cavity will, in addition, have couplers that will break these symmetries. However, in this study they are neglected as complete designs do not yet exist for them.

The ability to accurately model the crab cavities is essential in understanding their impact upon the stability of the machine. There are a number of approaches available to simulate the dynamics of the cavity's operating mode. The highest precision method available is direct numerical symplectic integration through the fields of the cavity. However, computationally such an approach is too slow for tracking $\sim 10^{5}$ turns of the LHC which are needed for longterm stability analysis. The need then is for an efficient representation that is precise and numerically stable. An efficient representation comes in the form of a transfer map 


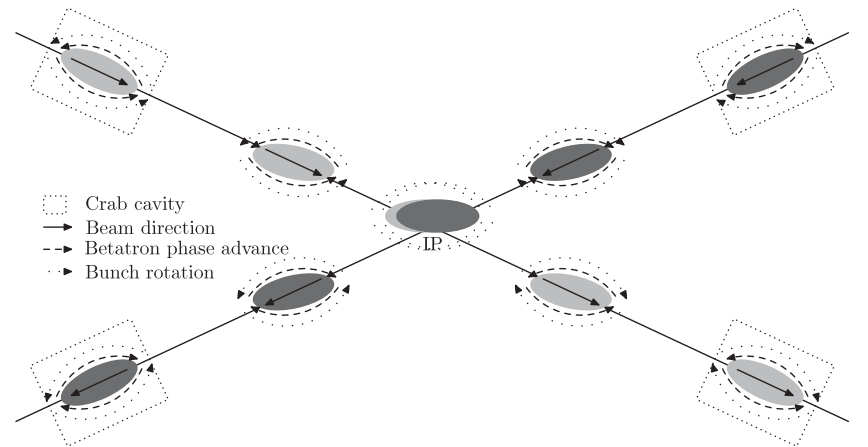

FIG. 1. Local crab crossing scheme showing creation of "crab bump."

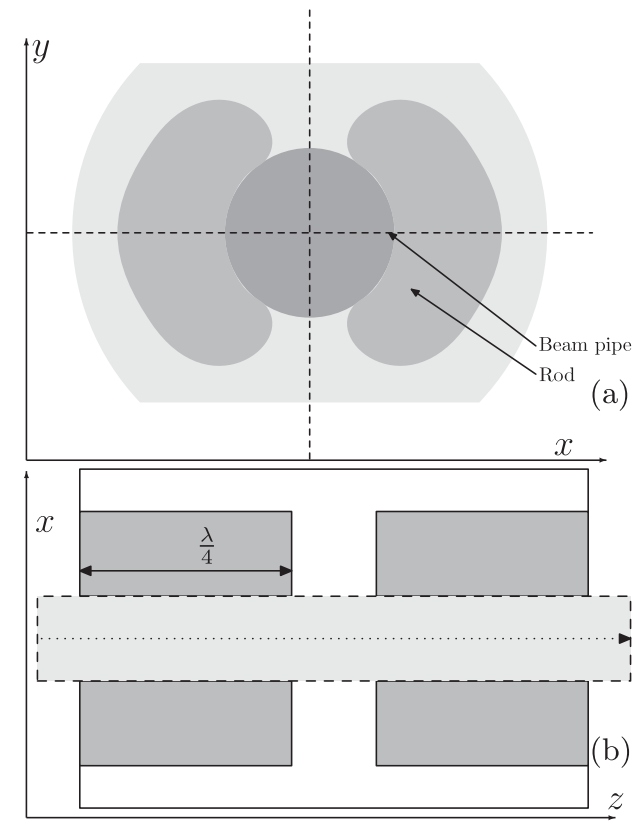

FIG. 2. Four rod cavity design (not to scale) with wavelength $\lambda$. The kick of the cavity shown is orientated in the $x$ plane, i.e., a horizontal crab cavity, projected in the (a) $x-y$ and (b) $z-x$ planes.

that describes the relationship between the canonical variables at the beginning and end of an accelerator element. A variety of approximations can be made in order to integrate the equations of motion to form such a transfer map prior to implementation for use in long-term tracking. A symplectic transfer map can be formed in the rigid particle (trajectory not affected through cavity) and axial (constant transverse position though the cavity) approximations, and further approximations can then be made to produce simpler models. One such implementation considers the expansion of the kick to be analogous to that of a magnet, giving a multipole representation [6,7].

Because of the uncertainty of the impact of the complete dynamics of a crab cavity on the HL-LHC, a more general approach, avoiding physical approximations, is considered here. The equations of motion, avoiding the rigid particle and axial approximations, cannot be solved exactly and a trade-off must be made in order to construct accurate transfer maps. Such a trade-off comes with a Taylor map, which is a transfer map described by a set of truncated power series of the initial variables. A Taylor map can be produced by using a differential algebra library [8], to integrate the equations of motion [9-11] through an analytical representation of the cavity's vector potential [11]. An analytical representation of the field is created by fitting the field data of the cavity to a general solution of the Helmholtz equation [11], from which an analytical vector potential can be found. The Taylor map presents a precise yet nonsymplectic approach to modelling the crab cavity due to the necessity to truncate the power series of the Taylor map. With increasing order the Taylor map tends towards the result of direct numerical integration. Symplectic error leads to errors in tracking which will accumulate turn by turn. In the past, considerations have been made to improve Taylor maps in terms of their symplecticity by use of symplectification algorithms $[12,13]$. These methods risk altering the dynamics of the model, removing the gain in precision expressed in the Taylor map [14]. For this reason they have not been considered.

The approach to understanding the relevant dynamics of the crab cavity with respect to the machine is to first construct a Taylor map of the crab cavity, thereby avoiding approximations made in other models. This process begins in Sec. II A in which an analytical representation of the fields is made. From this field representation, using a differential algebra code, a Taylor map is created in Sec. II B. In Sec. III a series of symplectic thin cavity models are developed from the accelerator Hamiltonian by applying a series of assumptions to simplify the description of the kick dependence on the spatial variables. The Taylor map is then used in Sec. III to make a direct comparison with the thin cavity models in order to consider the limitations of the assumptions applied by using them to calculate multipole coefficients. In Sec. IV, a number of thin cavity models are then compared to the Taylor map in the form of their impact upon the dynamic aperture. We show that by using Taylor maps to contain the detailed dynamics of a crab cavity we can determine the suitability and limitations of simpler symplectic models for long-term tracking. We also find the rf multipole and simple kick models are an adequate description of cavity dynamics for the case of HL-LHC.

\section{TAYLOR MAP MODEL}

\section{A. Field fitting}

Producing an analytical representation of the field allows for a Hamiltonian-based analysis of the particle dynamics of the operating mode. The analytical representation, compared with interpolation over a mesh of field data, allows for a more structured analysis of the fields, removing the necessity for a non-Maxwellian interpolation between 
mesh points. The field fitting method [11] assumes the vacuum form of Maxwell's equations is valid for a finite volume through the length of the cavity and that the field obeys the Helmholtz equation.

The longitudinal field may be expressed as an infinite series of cylindrical harmonic modes, where a boundary is chosen such that the harmonic functions describe the field within a cylinder running the length of the cavity. The field fitting method [11] expresses the field (and the associated vector potential) analytically, given explicit field data on the surface of the cylinder. Furthermore, it provides a way to compute the multipole coefficients of the crab cavity. Further details can be found in [11] and in Appendix A.

The series of functions needed to describe the field analytically $\left(\tilde{e}_{n}, \tilde{f}_{n}, \tilde{\alpha}_{n}\right.$, and $\left.\tilde{\beta}_{n}\right)$ are calculated from the field values on the surface of a cylinder of radius $R$ and knowledge of these functions gives access to the electric and magnetic fields for $\rho<R$. The field data which is used to populate the surface of the cylinder comes from an eigenmode solution to the cavity geometry produced using high frequency structure simulator (HFSS) [15]. The field data is produced on a specially defined cylindrical mesh of radius $R=20 \mathrm{~mm}$ with 16 azimuthal points and steps of $2 \mathrm{~mm}$ in $s$. Fourier transformations of the field data lead to the harmonic functions that describe the spatial component of the fields. An interpolation over a fine Cartesian mesh of field data is also produced to allow for validation of the fitting (denoted Interpolated).

Once these functions are established, the field is expressed in the form of cylindrical coordinates (denoted
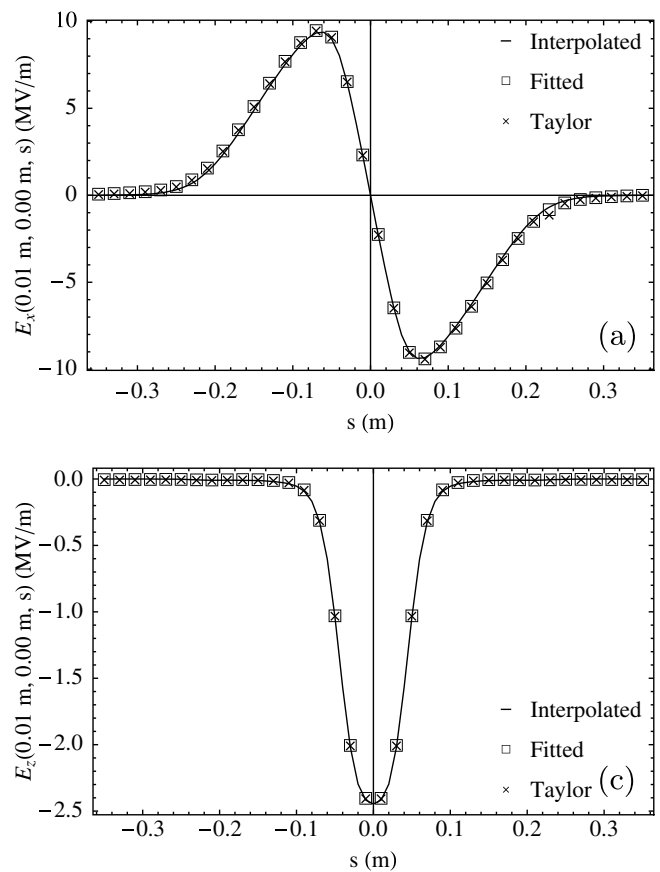

Fitted). This fitted field is truncated to $n=7$, with $n$ defined as per Eq. (A7), since beyond this only numerical noise is encountered. From this fitted analytical field the fields are converted to Cartesian coordinates, expanding to the form of a Taylor series in $x$ and $y$ (denoted Taylor), with coefficients defined as a function of the longitudinal position, $s$, as per the series given in Eq. (1). The Taylor form of the analytical fields is truncated to include fifthorder terms in the series of $x$ and $y$ for each discrete longitudinal position.

The field data of the four-rod cavity and the resulting analytical fields are shown in Fig. 3. The $E_{x}$ (plane of the kick) and $E_{z}$ are the most significant components of the electric field in the operating mode of the crab cavity. The $E_{x}$ component of the field's peaks coincide with the longitudinal tips of the rods. The $E_{z}$ component peaks at the longitudinal center of the cavity. The fields are dominated by the $n=1$ ("dipole") component, where $n$ is the azimuthal decomposition of the $E_{z}$ field such that $E_{z} \propto \cos (n \phi)$. In Fig. 3(b) an $E_{y}$ component of the field with a magnitude of $0.02 \%$ of $E_{x}$ is observed. However, from symmetry arguments such a field should not exist with the given cavity geometry. The dominant cause of this is the meshing noise in the eigenmode solver. Figure 3(d) shows that the $E_{z}$ field varies from the on axis field, which is of order $10^{7} \mathrm{~V} / \mathrm{m}$, with a transverse spatial dependence.

The fitting errors introduced by the two stages in the fitting process are shown in Fig. 4, by comparison with the meshed data. The electric field error between the Taylor and interpolated field with position in $x$, is shown in Fig. 4 .
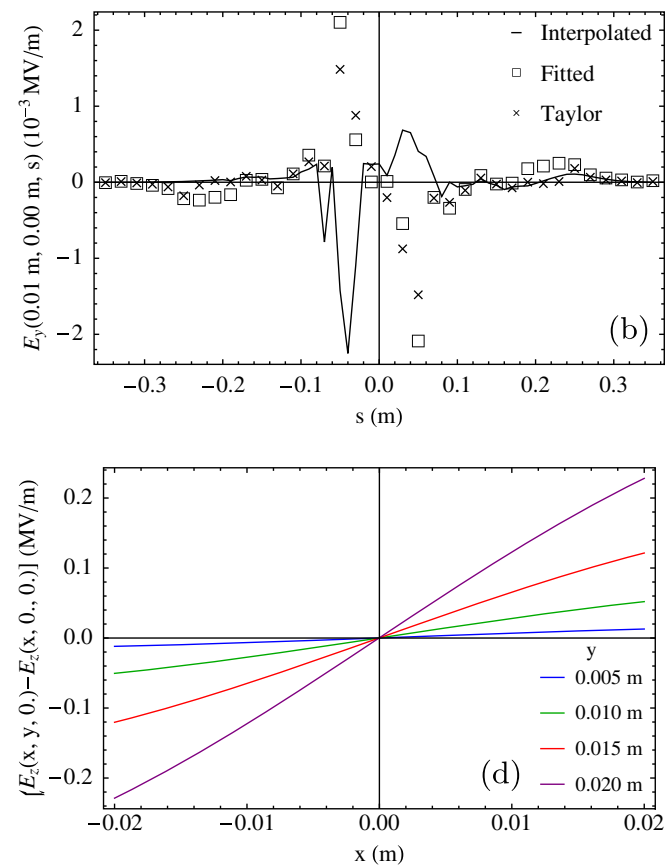

FIG. 3. E fields with varying $s$ at $x=0.01 \mathrm{~m}$ and $y=0.00 \mathrm{~m}$. (a) $E_{x}$, (b) $E_{y}$, and (c) $E_{z}$. The Interpolated mesh fields and Fitted and Taylor analytical representations are shown. (d) $E_{z}$ transverse variation in $x$ and $y$ away from variation at $y=0 \mathrm{~m}$ from Taylor field. 

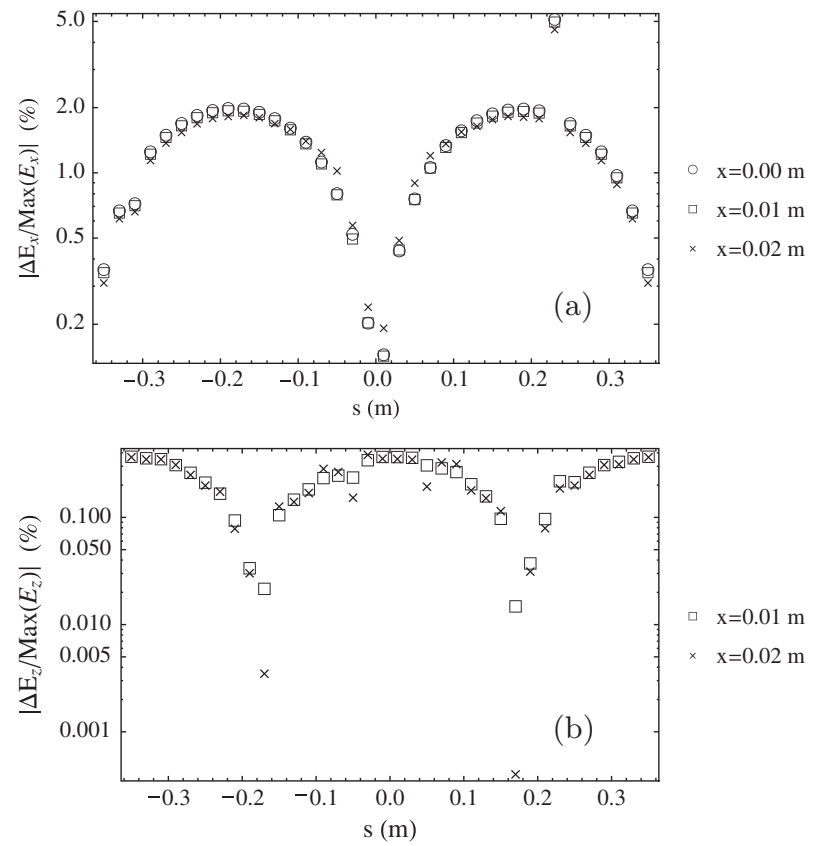

FIG. 4. $E$ fitting error with varying $x$. Difference between interpolated and Taylor fitted fields; (a) $E_{x}$ and (b) $E_{z}$.

The error is normalized to the peak field value at the given $x$ value along the whole length of the cavity. The error in $E_{z}$ has a maximum error of $0.3 \%$ of the peak field value.

To study the variation in the field with radius requires the introduction of generalized gradient functions [11,16-18]. These functions allow the field to be discretized in terms of components with a given azimuthal dependency and radial power. This discretization in terms of radial power was not available in Eqs. (A7), (A8), and (A9). Hence the longitudinal field can be written

$E_{z}(x, y, s)=\sum_{m=0}^{M} \sum_{n=1}^{N} \frac{1}{2^{n+2 m} m !(n+m) !}\left(x^{2}+y^{2}\right)^{m}$

$\left(\Re\left[(x+\mathrm{i} y)^{n}\right] C_{z c}^{(l)}(s, n, m)+\Im\left[(x+\mathrm{i} y)^{n}\right] C_{z s}^{(l)}(s, n, m)\right)$,

where the gradient functions are defined in terms of the harmonic functions,

$$
\begin{aligned}
& C_{z c}^{(l)}(s, n, m) \\
& =\int_{-\infty}^{\infty} \frac{d k}{\sqrt{2 \pi}} e^{\mathrm{i} k s} \operatorname{sgn}\left(k^{2}-k_{l}^{2}\right)^{m} \kappa_{l}(k)^{n+2 m} \tilde{e}_{n}(k), \\
& C_{z s}^{(l)}(s, n, m) \\
& \quad=\int_{-\infty}^{\infty} \frac{d k}{\sqrt{2 \pi}} e^{\mathrm{i} k s} \operatorname{sgn}\left(k^{2}-k_{l}^{2}\right)^{m} \kappa_{l}(k)^{n+2 m} \tilde{f}_{n}(k) .
\end{aligned}
$$

Considering the longitudinal field a normal gradient function $C_{z c}$ and skew $C_{z s}$ are required to describe the field, by the transformation of Eq. (A9) into Cartesian coordinates.

\section{B. Taylor maps from integration of field}

The Taylor maps are produced using an explicit symplectic integrator developed by $\mathrm{Wu}$, Forest, and Robin [19] (WFR) implemented in COSY infinity [8]. The WFR integrator was developed for an $s$-dependent Hamiltonian for charged particles moving through an electromagnetic field. Two implementations of this integrator are considered, one numerical and another using differential algebra. Some details of the calculations are shown in Appendix B and the resulting degree of deviation from symplecticity is discussed in Appendix C.

To calculate a Taylor map the numerical integration must be carried out using a differential algebra library [8], which considers all variables as a truncated power series. Two levels of truncation occur in a differential algebra library: The coefficient value and the maximum order of the sum of exponents, shown in Eq. (B4). The WFR integrator [19] is used to integrate through the Taylor analytic vector potential with mathematical operations performed directly upon the individual terms of the vector potential series. The integration is performed with unassigned initial variables using COSY infinity [8], truncating the sum of exponents to eighth order. Higher order integrators can be determined from the expansion defined in [20] using the Lie transformations of the WFR integrator [19].

The choice of step size and truncation order have been studied, balancing computing time with precision. The variation of momentum kick as a function of step size is shown in Fig. 5, indicating that the variation rapidly decreases with step size. The chosen step size was $0.01 \mathrm{~m}$, giving 70 steps through the cavity. This choice gives an error at the level of $10^{-10}$ of the size of the peak $p_{y}$ kick. To reduce the variation in the kick to less than $0.01 \%$ of this error required a reduction in the step size of greater than a factor of 2 .

In general, with increasing order of exponents there is a decreasing contribution from all the terms of that order.

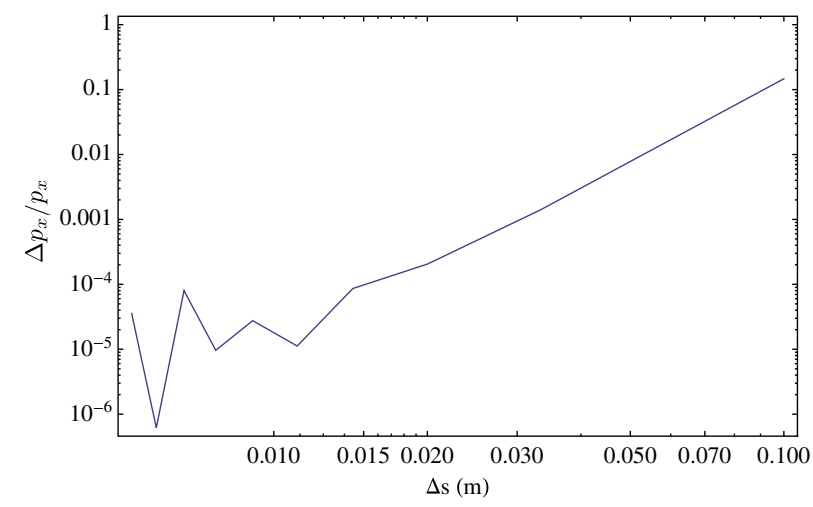

FIG. 5. Convergence of integration. Final $p_{x}$ error produced with WFR integration with varying step size compared with integration with step size $0.5 \mathrm{~mm}$. Initial conditions $\left(x, p_{x}, y, p_{y}, \delta\right)=0$, $z=0.01$. 
The order of truncation is chosen to allow terms up to $\rho^{5}$ transversely in combination with a time-dependent component with a minimum of third order. This results in the sum of exponents truncated to eighth order. The spatial component truncation was justified from the field fitting from which it was found that higher order terms contributed at the level of mesh noise. The time component truncation for the fifth order in $x$ and $y$ terms of the vector potential equates to an error of $0.03 \%$ at $z=5 \mathrm{~cm}$ compared with using a full sine function. Furthermore, increasing the order significantly more than 8 creates many more terms that would need to be applied in the tracking code, making it computationally slower.

The numerical precision was chosen to be $10^{-30}$. Given that a double numerical precision number can have up to 14 significant figures and that the largest Taylor map coefficients are equal to one, any term which contributes at the level of $10^{-28} \%$ of these largest terms will not have a visible effect at the level of double precision. This means that at this numerical truncation there is no risk of losing terms which will contribute to a double precision result.

\section{THIN MODELS}

In order to produce an $s$-independent model of the crab cavity a series of approximations must be applied in order to split the Hamiltonian into drift and kick components. From the rigid particle approximation (trajectory not affected through cavity), axial approximation (constant transverse position though the cavity), and the PanofskyWenzel theorem [21] (constant velocity through the cavity and zero impact from $A_{x, y}$ ), the Hamiltonian can be split into a drift and kick component, where $H_{\text {kick }}(x, y, z)$ is only dependent on the spatial coordinates and defined in terms of the averaged longitudinal component of the vector potential by

$$
\begin{aligned}
H_{\text {kick }} & =-\frac{e_{0}}{p_{0}} \frac{1}{L} \int_{0}^{L} A_{z}\left(x, y, z, t=s /\left(\beta_{0} c\right)-z / c\right) d s \\
& =-\frac{1}{L} \int_{0}^{L} a_{z}\left(x, y, z, t=s /\left(\beta_{0} c\right)-z / c\right) d s,
\end{aligned}
$$

where $t=s /\left(\beta_{0} c\right)-z / c$ in the delay in the arrival time of the particle with respect to the reference particle (see Appendix B). The drift component is only dependent on momentum; therefore, both components of the Hamiltonian are exactly solvable with Hamilton's equations and lead to symplectic transfer maps. A number of further approximations can be applied to Eq. (4) in order to simplify the dynamics contained within the model. Three different models were considered in this thin lens approximation in order to compare with the dynamics contained within the Taylor map.

In its most general form the vector potential can be expressed in terms of the $E_{z}^{(l)}$ defined in Eq. (A9). In terms of the generalized gradients in Eq. (1) (which are directly calculable from the field surface data), in Cartesian coordinates the integrated $A_{z}$ is given by

$$
\begin{aligned}
\int_{0}^{L} A_{z} \mathrm{~d} s & =\Re\left[\sum_{l}-\frac{\mathrm{i}}{\omega_{l}} e^{-\mathrm{i}\left(-k_{l} z+\Phi_{l}\right)} \int_{0}^{L} e^{-\mathrm{i} k_{l} s / \beta_{0}} E_{z}^{(l)} \mathrm{d} s\right]=\Re\left[\sum_{l}-\frac{\mathrm{i}}{\omega_{l}} e^{-\mathrm{i}\left(-k_{l} z+\Phi_{l}\right)}\right. \\
& \left.\times \sum_{m=0}^{M} \sum_{n=1}^{N} \frac{\left(x^{2}+y^{2}\right)^{m}}{2^{n+2 m} m !(n+m) !}\left\{\Re\left[(x+\mathrm{i} y)^{n}\right] \int_{0}^{L} e^{\mathrm{i} k_{l} s / \beta_{0}} C_{z c}^{(l)}(s, n, m) \mathrm{d} s+\Im\left[(x+\mathrm{i} y)^{n}\right] \int_{0}^{L} e^{\mathrm{i} k_{l} s / \beta_{0}} C_{z s}^{(l)}(s, n, m) \mathrm{d} s\right\}\right]
\end{aligned}
$$

for multiple standing modes. This integrated vector potential in Eq. (5) gives the generalized rf multipole kick model. By applying Hamilton's equations to Eq. (4) the following momentum kicks are found [suppressing the superscript (l) and assuming a singular standing mode]:

$$
\begin{aligned}
& p_{x} \mapsto p_{x}+\frac{e_{0}}{p_{0}} \sin \left(\frac{\omega z}{c}-\Phi\right) \sum_{m=0}^{M} \sum_{n=1}^{N}\left\{\left(x^{2}+y^{2}\right)^{m}\left(\Re\left[(x+\mathrm{i} y)^{n-1}\right] b_{n, m}+\Im\left[(x+\mathrm{i} y)^{n-1}\right] a_{n, m}\right)\right. \\
&\left.+2 \frac{m}{n} x\left(x^{2}+y^{2}\right)^{m-1}\left(\Re\left[(x+\mathrm{i} y)^{n}\right] b_{n, m}+\Im\left[(x+\mathrm{i} y)^{n}\right] a_{n, m}\right)\right\}, \\
& p_{y} \mapsto p_{y}-\frac{e_{0}}{p_{0}} \sin \left(\frac{\omega z}{c}-\Phi\right) \sum_{m=0}^{M} \sum_{n=1}^{N}\left\{\left(x^{2}+y^{2}\right)^{m}\left(\Re\left[\mathrm{i}(x+\mathrm{i} y)^{n-1}\right] b_{n, m}+\Im\left[\mathrm{i}(x+\mathrm{i} y)^{n-1}\right] a_{n, m}\right)\right. \\
&\left.+2 \frac{m}{n} y\left(x^{2}+y^{2}\right)^{m-1}\left(\Re\left[(x+\mathrm{i} y)^{n}\right] b_{n, m}+\Im\left[(x+\mathrm{i} y)^{n}\right] a_{n, m}\right)\right\}, \\
& \delta \mapsto \delta-\frac{e_{0}}{p_{0}} \frac{\omega}{c} \cos \left(\frac{\omega z}{c}-\Phi\right) \sum_{m=0}^{M} \sum_{n=1}^{N} \frac{1}{n}\left(x^{2}+y^{2}\right)^{m}\left(\Re\left[(x+\mathrm{i} y)^{n}\right] b_{n, m}+\Im\left[(x+\mathrm{i} y)^{n}\right] a_{n, m}\right),
\end{aligned}
$$


where the generalized multipole coefficients are defined by

$$
\begin{aligned}
& b_{n, m}=\Re\left[\frac{n}{\omega 2^{n+2 m} m !(n+m) ! n L} \int_{0}^{L} e^{-\mathrm{i} \frac{\omega s / \beta_{0}}{c}} C_{z c}(s, n, m) \mathrm{d} s\right], \\
& a_{n, m}=\Re\left[\frac{n}{\omega 2^{n+2 m} m !(n+m) ! L} \int_{0}^{L} e^{-\mathrm{i} \frac{\omega s / \beta_{0}}{c}} C_{z s}(s, n, m) \mathrm{d} s\right] .
\end{aligned}
$$

The only approximations applied to the Hamiltonian in this generalized rf multipole kick model are the ones leading to Eq. (4) [21].

A simplification on the generalized rf multipole kick model can be found from considering the energy transferred to a particle from a crab cavity. In [6] it is stated that the voltage $V$ experienced by a charged particle obeys the following relation:

$$
\nabla^{2} V-\left(\frac{k_{l}}{\gamma_{0}}\right)^{2} V=0
$$

where the voltage is given by

$$
V(x, y)=\int_{0}^{L} E_{z}(x, y, s) d s,
$$

in the axial and rigid particle approximations and for vanishing fields at the boundaries.

In the limit that $k_{l} / \gamma_{0} \rightarrow 0[6]$, the integrated vector potential takes the form of a solution to the Laplace equation, resulting in the following integrated vector potential $[6,7,22]$ :

$$
\begin{aligned}
\int_{0}^{L} A_{z} \mathrm{~d} s & =\Re\left[e^{\mathrm{i}\left(k_{l} z+\Phi_{l}\right)} \int_{0}^{L} A_{z}^{(l)} \mathrm{d} s\right] \\
& =\Re\left[e^{\mathrm{i}\left(k_{l} z-\Phi_{l}\right)} \sum_{n=1}^{N} \frac{\rho^{n}}{n}\left(b_{n} \cos (n \phi)+a_{n} \sin (n \phi)\right)\right],
\end{aligned}
$$

where $a_{n}$ and $b_{n}$ are the skew and normal rf-multipole coefficients of a spatial component of a particular standing wave mode, and $A_{z}^{(l)}$ is the spatial component of the longitudinal vector potential. In $[7,22]$ the convention was to have complex rf-multipole coefficients $\left(b_{n}^{\text {[complex convention] }}, a_{n}^{\text {[complex convention] }}\right)$ with their argument representing the phase rather than having a separate phase variable such that,

$$
\begin{aligned}
& b_{n}^{[\text {complex convention }]}=b_{n} e^{\mathrm{i} \Phi_{l}}, \\
& a_{n}^{[\text {complex convention }]}=a_{n} e^{\mathrm{i} \Phi_{l}} .
\end{aligned}
$$

The rf-multipole coefficients directly equate to the generalized multipoles, in Eq. (7), by the relation $b_{n}=b_{n, 0}$. From the Hamiltonian given in Eq. (4) the equations of motion, in Cartesian coordinates, are given by the series of kicks $[6,7]$,

$$
\begin{aligned}
p_{x} & \mapsto p_{x}+\frac{e_{0}}{p_{0}} \sin \left(\frac{\omega z}{c}+\Phi\right) \sum_{n=1}^{N} \Re\left[\left(b_{n}+\mathrm{i} a_{n}\right)(x+\mathrm{i} y)^{n-1}\right], \\
p_{y} & \mapsto p_{y}-\frac{e_{0}}{p_{0}} \sin \left(\frac{\omega z}{c}+\Phi\right) \sum_{n=1}^{N} \Im\left[\left(b_{n}+\mathrm{i} a_{n}\right)(x+\mathrm{i} y)^{n-1}\right], \\
\delta & \mapsto \delta+\frac{e_{0}}{p_{0}} \frac{\omega}{c} \cos \left(\frac{\omega z}{c}+\Phi\right) \sum_{n=1}^{N} \frac{1}{n} \Re\left[\left(b_{n}+\mathrm{i} a_{n}\right)(x+\mathrm{i} y)^{n}\right] .
\end{aligned}
$$

This model is called the rf-multipole kick model.

A further simplification on the rf-multipole kick model can be made by selecting only one multipole component to represent the cavity. This model is called the simple kick model. For the case of a crab cavity this is performed by only considering the kick resulting from the $b_{1}$ coefficient [3].

The multipole coefficients can be calculated by a variety of methods. Each method decomposes the linearly integrated momentum kick azimuthally directly from the cavity's fields. This makes the assumption that the radial dependence for each azimuthal component is $\rho^{n-1}$, where $n$ is the azimuthal index. The values of the multipole coefficients were presented previously and use the same meshed data as that used for this study [7]; they are shown in Table I. They were calculated by using the PanofskyWenzel (PW) method [21] at a $20 \mathrm{~mm}$ radius, using the same mesh data as used for the field fitting.

While Taylor maps introduce symplectic error, it is still possible to compare them in terms of their single pass dynamics with the rf multipoles and generalized rf multipoles. The rf multipole model makes a number of assumptions on the dynamics that are not included in the Taylor maps. The first is that trajectory of the particle, within the cavity, has little impact upon the momentum kicks received i.e., the axial approximation. The second is the that the particles are rigid and the third is that there is zero impact from $A_{x, y}$ [21]. The fourth assumption leads to the removal of terms with $m>0$ from Eq. (1) in the limit of $k / \gamma_{0} \rightarrow 0$ [6].

\section{A. Radio frequency multipole kick model and Taylor map comparison}

To directly compare the Taylor map with the rf-multipole model, multipole coefficients can be extracted from the Taylor map. The following method can only be applied in the case where only a single standing mode is contained within the Taylor map. Further modes would result in each Taylor map term containing the sum of the terms from each mode, therefore making the extraction of the effective multipole coefficients difficult. In order to perform this 
TABLE I. Comparison of multipole coefficients calculated from the Taylor map, compared with the Helmholtz decomposition and Panofsky-Wenzel methods at $\rho=20 \mathrm{~mm}$ [22]. Coefficients in units of $\mathrm{mT} / \mathrm{m}^{n-1}$ normalized to $10 \mathrm{MV}$.

\begin{tabular}{lccrr}
\hline \hline Multipole coefficient & Terms used & Taylor map & Helmholtz decomposition & Panofsky-Wenzel \\
\hline$b_{1}$ & $p_{x}(z)$ & $3.33 \times 10^{+1}$ & $3.33 \times 10^{+1}$ & $3.33 \times 10^{+1}$ \\
$b_{2}$ & $p_{x}(x z)$ & $7.36 \times 10^{-2}$ & $7.39 \times 10^{-2}$ & $-3.00 \times 10^{-2}$ \\
$a_{2}$ & $p_{x}(y z)$ & $-3.31 \times 10^{-2}$ & $-3.31 \times 10^{-2}$ & $-1.40 \times 10^{-3}$ \\
$b_{3}$ & $p_{x}\left(x^{2} z\right)$ & $9.06 \times 10^{+2}$ & $9.06 \times 10^{+2}$ & $8.98 \times 10^{+2}$ \\
$a_{3}$ & $p_{x}(x y z)$ & -1.85 & $-0.92 \times 10^{-1}$ & $-2.40 \times 10^{-1}$ \\
$b_{4}$ & $p_{x}\left(x^{3} z\right)$ & 3.07 & 2.57 & $3.90 \times 10^{+1}$ \\
$a_{4}$ & $p_{x}\left(x^{2} y z\right)$ & $6.11 \times 10^{+2}$ & $2.033 \times 10^{+2}$ & $1.20 \times 10^{+1}$ \\
$b_{5}$ & $p_{x}\left(x^{4} z\right)$ & $-2.40 \times 10^{+6}$ & $-2.40 \times 10^{+6}$ & $-2.39 \times 10^{+6}$ \\
$a_{5}$ & $p_{x}\left(x^{3} y z\right)$ & $-5.95 \times 10^{+4}$ & $-1.49 \times 10^{+4}$ & $-9.27 \times 10^{+2}$ \\
\hline \hline
\end{tabular}

comparison the transverse rf-multipole kicks, as described in Eq. (12), undergo a Taylor series expansion for one particular mode,

$$
p_{x} \mapsto p_{x}+\frac{e_{0}}{p_{0}} b_{1} \frac{\omega}{c} z+\frac{e_{0}}{p_{0}} b_{2} \frac{\omega}{c} x z+\frac{e_{0}}{p_{0}} b_{3} \frac{\omega}{c} x^{2} z+\cdots
$$

Terms in the Taylor map share the same dependencies upon the canonical variables as those given by the Taylor expanded kick terms of the rf multipoles. These power series terms are directly compared in terms of their coefficients, allowing an effective multipole strength to be found from each term of the power series.

The dynamics as described by the generalized rf-multipole kick model in Eq. (13) indicates that multiple generalized multipoles contribute to a single term in the Taylor map. This implies there should not be a one-to-one mapping between the rf-multipole coefficients and the Taylor map if the generalized multipoles for $m>0$ are significant. The multipole coefficients from terms of the $p_{x}$ kick with the largest $x$ exponent in the Taylor series expansion are compared in Table I, with the results of the PW and Helmholtz decomposition (HD) methods [22]; evaluations were done at a radius of $20 \mathrm{~mm}$. More detail of the multipole calculations by the PW and HD methods can be found in [22]. In the table it can be seen that there are skew components and even normal components that result from numerical errors in the field data. These form as a result of the field map errors seen in Sec. II A. The dominant multipole coefficients $\left(b_{1}, b_{3}\right.$, and $\left.b_{5}\right)$ show agreement to three significant figures between the Helmholtz decomposition and extracted Taylor map values. This suggests to this level of precision that the generalized rf multipoles for $m>0$ do not contribute significantly to the kick. There are significant differences between the PW and HD that arise from the first-order radial approximation used in the Panofsky-Wenzel method [22]. Table I therefore shows that the transverse dependence of the kicks is more sensitive to the multipole calculation method than the generalized rf multipoles for $m>0$.

\section{B. Generalized rf-multipole kick model and Taylor map comparison}

The impact of the assumption $k_{l} / \gamma_{0} \rightarrow 0$ can be seen by calculating generalized rf multipoles from the Taylor map. By a similar method to Sec. III A, a Taylor series expansion is performed of the momentum kicks generated in the generalized rf-multipole kick model from Eq. (6) resulting in a set of simultaneous equations from which the generalized multipoles can be calculated. For example, by considering the terms in which the $b_{3,0}$ multipole coefficient appears,

$$
\begin{aligned}
& p_{x}\left(x^{2} z\right)=\frac{e_{0}}{p_{0}} \frac{\omega}{c}\left(b_{3,0}+3 b_{1,1}\right), \\
& p_{x}\left(y^{2} z\right)=-\frac{e_{0}}{p_{0}} \frac{\omega}{c}\left(b_{3,0}-b_{1,1}\right),
\end{aligned}
$$

where $p_{x}\left(x^{2} z\right)$ is the $p_{x}$ Taylor map series coefficient corresponding to the $x^{2} z$ term, the coefficients $b_{1,1}$ and $b_{3,0}$ can be calculated. The results of the generalized multipoles contributing to the terms used to calculate the $b_{1}, b_{3}$, and $b_{5}$ from the Taylor map are shown in Table II. These are compared with those directly calculated from the generalized gradients (field fitting). In Table II the scaled value shows the contribution of the different generalized rf multipoles as a fraction of the kick from the $b_{1}$ component evaluated at $x=20 \mathrm{~mm}$. From the scaled values in Table II it can be seen that the generalized rf multipoles for $m>0$ provide a maximum contribution of order $0.001 \%$ of the $b_{1}$ kick. By contrast the rf multipoles for $n>1$ contribute of order $1 \%$. It is also evident that these higher order kicks do not agree between the Taylor map and field fitting. This suggests that other dynamics beyond that of the rf-multipole models contributes at this level of sensitivity. This means that at this level of sensitivity the assumptions made to form the thin cavity models start to fail.

\section{DYNAMIC APERTURE}

In order to perform dynamic aperture studies of the HL-LHC the kick code SixTrack was used [23,24]. 
TABLE II. Generalized multipole coefficients calculated from the Taylor map and generalized gradients. Coefficients in units of $\mathrm{mT} / \mathrm{m}^{n+2 m-1}$ for $b_{n, m}$, normalized to $10 \mathrm{MV}$. Where the scaled value is given by $\left(b_{n, m} / b_{1,0}\right) \times(20 \mathrm{~mm})^{n+2 m-1}$.

\begin{tabular}{|c|c|c|c|c|c|}
\hline \multirow{2}{*}{$\begin{array}{l}\text { Multipole } \\
\text { coefficient }\end{array}$} & \multirow[b]{2}{*}{ Terms used } & \multicolumn{2}{|c|}{ Taylor map } & \multicolumn{2}{|c|}{ Field fitting } \\
\hline & & Multipole value & Scaled value $\left[10^{-4}\right]$ & Multipole value & Scaled value $\left[10^{-4}\right]$ \\
\hline$b_{1,0}$ & $p_{x}(z)$ & 33.333 & 10000.000 & 33.333 & 10000.000 \\
\hline$b_{1,1}$ & $p_{x}\left(x^{2} z\right), p_{x}\left(y^{2} z\right)$ & -0.146 & -0.017 & -0.438 & -0.052 \\
\hline$b_{1,2}^{1,2}$ & $p_{x}\left(x^{4} z\right), p_{x}\left(y^{4} z\right), p_{y}\left(x^{3} y z\right)$ & 668.523 & 0.032 & -123.657 & -0.006 \\
\hline$b_{3,0}$ & $p_{x}\left(x^{2} z\right), p_{x}\left(y^{2} z\right)$ & 905.977 & 108.717 & 906.178 & 108.741 \\
\hline$b_{3,1}$ & $p_{x}\left(x^{4} z\right), p_{x}\left(y^{4} z\right), p_{y}\left(x^{3} y z\right)$ & -121.248 & -0.006 & -221.112 & -0.011 \\
\hline$b_{5,0}$ & $p_{x}\left(x^{4} z\right), p_{x}\left(y^{4} z\right), p_{y}\left(x^{3} y z\right)$ & $-2.399 \times 10^{+6}$ & -115.152 & $-2.399 \times 10^{+6}$ & -115.152 \\
\hline
\end{tabular}

SixTrack, being a kick code, uses thin kick models to represent accelerator elements. To implement the thick Taylor maps, while conserving the use of the drift and kick model contained in SixTrack, antidrifts were introduced. These antidrifts were produced in the form of Taylor maps using COSY infinity to integrate in reverse for half the length of the cavity. By sandwiching the Taylor map between two antidrifts, as shown in Fig. 6, the Taylor maps behaved as a kick and orbit displacement.

The dynamic aperture (DA) study is performed taking the minimum value from 60 variants of the machine with varying magnet multipole errors from the arc dipoles and quadrupoles [23,24], across 19 values of $\arctan \left(\varepsilon_{x} / \varepsilon_{y}\right)$ between $0^{\circ}$ and $90^{\circ}$, where $\varepsilon_{x, y}$ are the single particle emittances. The momentum offset of the initial conditions is set to the momentum corresponding to $3 / 4$ of the bucket half-height. The study is performed on the SLHCV3.1b optics [25] with $\beta_{x, y}^{*}=15 \mathrm{~cm}$ at IP1 and IP5. For this study the addition of beam-beam interaction and insertion region magnet errors is not considered. Studies with the insertion region magnet errors using the rf-multipole kick model up to $n=4$ can be found in [22]. The DA is defined by the mean amplitude given in units of the root mean square width of a bunch $(\sigma)$. The error on the DA is given by the precision error at that particular angle defined by the step size used in the search for the DA.

An eighth order map is required to contain all the relevant dynamical features to the crab cavity and such a

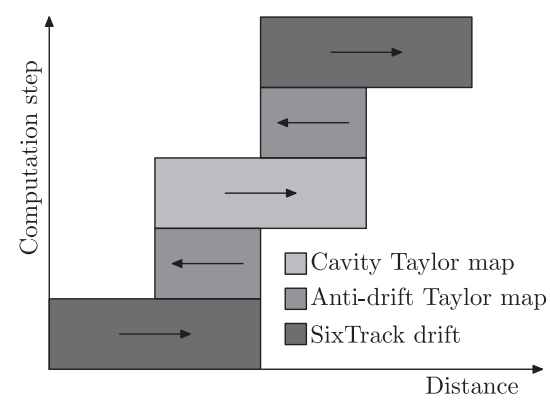

FIG. 6. The computation steps taken to pass through one crab cavity in SixTrack to allow for a Taylor map to work in a thin tracking code. map equates to having power series with up to 3003 terms. However, many of these terms have a very small contribution to the final result of the evaluated map. The reduced coefficient maps (RCMs) are an attempt to remove terms with limited impact on the dynamics, resulting in a more compact representation and an associated increase in symplectic error. A tracking-based study was performed to identify the 500 most significant terms in the eighth order map while monitoring symplectic error in the motion.

In Fig. 7 the DA is presented for four different cavity models. The Taylor map used was a RCM with 500 coefficients per series and this is compared with three different thin lens models: The simple kick model considers only the kick from $b_{1}$, the rf-multipole kick model from HD multipoles (RFM-HD), and rf-multipole kick model from PW multipoles (RFM-PW). Comparing the difference between the Taylor map and RFM-HD there is a maximum difference of $0.4 \sigma$. This difference may arise from a combination of symplectic error and additional dynamics as observed in Table II. By contrast comparing the simple kick model with the Taylor map there is a maximum difference of $0.8 \sigma$. Furthermore there is a maximum

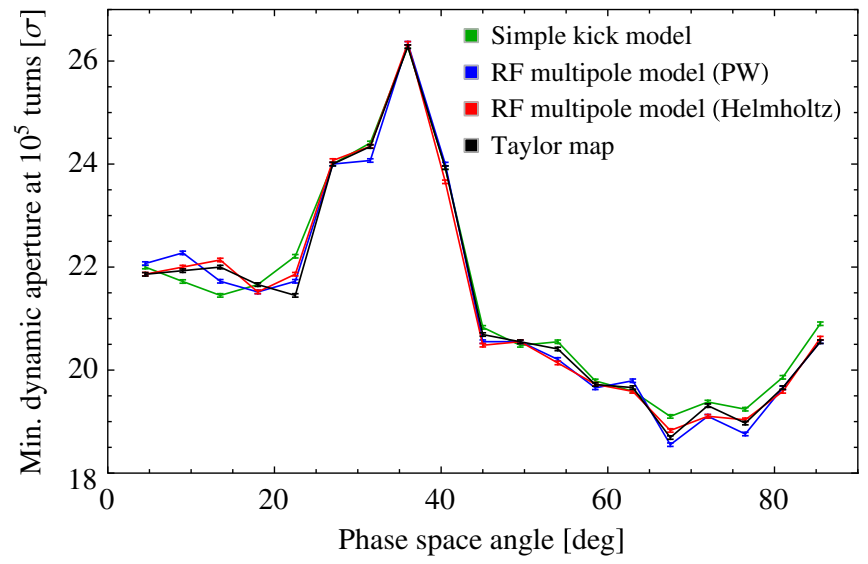

FIG. 7. Impact of different crab cavity models on the minimum dynamic aperture after $10^{5}$ turns, comparing the 500 coefficient Taylor map with the rf-multipole kick model (using multipoles calculated using the Helmholtz and PW decompositions) and the simple kick model. 
difference between the RFM-PW and RFM-HD models of $0.4 \sigma$. This suggests that the sensitivity to additional dynamics and symplectic error is of order of the sensitivity to the multipole calculation, and the various models are consistent. This would indicate that the rf multipole kick model gives a good approximation to the crab cavity dynamics relevant to the HL-LHC given the tolerances in the calculation of the multipole coefficients.

\section{CONCLUSION}

Dynamic aperture calculations with crab cavities are important for the HL-LHC design studies as they have not been used in a hadron collider before. Because of the sensitivity of a hadron machine to nonlinearities it is important to consider all the possible effects that might arise from crab cavities. In order to include all possible effects, accurate models of the crab cavities were developed in this work.

By using Taylor maps to contain the detailed dynamics of a crab cavity, we show that we can develop an understanding of the suitability and limitations of simpler symplectic models. It has been shown that, in their construction, rf multipoles contain the dominant dependencies of the $p_{x}$ and $p_{y}$ kicks found in the Taylor map. Furthermore, despite the tracking uncertainty from symplectic error, Taylor maps have been successfully applied in the calculation of dynamic aperture and have been used to show that the rf multipole description makes improvements over the simple kick model in describing the resulting variation in dynamic aperture of the HL-LHC lattice. Although the effect on dynamic aperture of the crab cavities is relatively small [22] it has been shown that the dynamic aperture's sensitivity to dynamics beyond the rf-multipole kick model is less than the sensitivity to the details of the rfmultipole calculation. The rf-multipole model, however, has been shown, using the Taylor map, that it does not contain the full dynamic description of the crab cavities. Furthermore, the dynamics beyond the rf-multipole description cannot be described in the generalized rfmultipole model. Note that the computed minimum DA is slightly larger than the fitted field reference radius, due to a common field map between the Taylor map and the multipole studies, but this extension is small and is not expected to significantly impact the accuracy of the method.

Overall the dynamic aperture study performed using the Taylor maps validates the simple kick (or thin) model and indicates that for the crab cavities in the LHC upgrade scenarios, the rf-multipole and simple kick models are an adequate description of cavity dynamics. A full study of the impact of noise, particularly experimental data noise, would be very interesting and a very good topic for future work. We hope the models presented and developed are useful for modeling complex time-dependent elements in future machines.

\section{ACKNOWLEDGMENTS}

The authors would like to acknowledge Frank Schmidt for advice regarding SixTrack, María Navarro and Alexej Grudiev for communication of cavity field data, and Massimo Giovannozi for advice regarding symplecticity and careful reading of the manuscript. Research supported by EU FP7 HiLumi LHC, Grant Agreement No. 284404.

\section{APPENDIX A: FIELD FITTING TECHNIQUES}

The electric field $\vec{E}$ contained within the vacuum of a rf cavity satisfies the wave equation,

$$
\nabla^{2} \vec{E}-\frac{1}{c^{2}} \frac{\partial^{2} \vec{E}}{\partial t^{2}}=0
$$

It is assumed that for a standing wave mode in a cavity, the $\vec{E}$ field has a harmonic time dependence, and the spatial and time dependent field components are separable,

$$
\vec{E}(\mathbf{r}, t)=\Re\left[\sum_{l} \vec{E}^{(l)}(\mathbf{r}) e^{-\mathrm{i}\left(\omega_{l} t+\Phi_{l}\right)}\right],
$$

where $\omega_{l}$ and $\Phi_{l}$ are the frequency and phase of harmonic time dependence of a given mode. The spatial component of the field obeys the vector Helmholtz equation,

$$
\nabla^{2} \vec{E}^{(l)}+k_{l}^{2} \vec{E}^{(l)}=0
$$

where $k_{l} \equiv \omega_{l} / c$, where the fields can be related to the vector potential through the expressions

$$
\begin{aligned}
\vec{E} & =-\frac{\partial \vec{A}}{\partial t}, \\
\vec{B} & =\nabla \times \vec{A} .
\end{aligned}
$$

The gauge is chosen such that the scalar potential is zero, such that by assuming standing wave modes the vector potential is directly related to the spatial mode of the electric field, $\vec{E}^{(l)}$, such that,

$$
\vec{A}=\Re\left[\sum_{l}-\frac{\mathrm{i}}{\omega_{l}} \vec{E}^{(l)} e^{-\mathrm{i}\left(\omega_{l} t+\Phi_{l}\right)}\right],
$$

which removes the necessity to consider the $\vec{B}$ field component. The general solution to the Helmholtz equation in cylindrical coordinates $(\rho, \phi, z)$ can be written as [11] [for the remainder of this section the superscript (l) is dropped, discussing only the spatial component of a given mode] 


$$
\begin{gathered}
E_{r}(\mathbf{r})=\int_{-\infty}^{\infty} \frac{d k}{\sqrt{2 \pi}} e^{\mathrm{i} k z}\left(\frac{-\mathrm{i} k}{\kappa_{l}}\right)\left\{\tilde{e}_{0} R_{1}(k, \rho)+\sum_{n=1}^{\infty}\left[\left(\tilde{e}_{n}(k) R_{n+1}(k, \rho)+\tilde{\beta}_{n}(k) \frac{R_{n}(k, \rho)}{\kappa_{l} \rho}\right) \cos (n \phi)\right.\right. \\
\left.\left.+\left(\tilde{f}_{n}(k) R_{n+1}(k, \rho)+\tilde{\alpha}_{n}(k) \frac{R_{n}(k, \rho)}{\kappa_{l} \rho}\right) \sin (n \phi)\right]\right\}, \\
E_{\phi}(\mathbf{r})=\int_{-\infty}^{\infty} \frac{d k}{\sqrt{2 \pi}} e^{\mathrm{i} k z}\left(\frac{\mathrm{i} k}{\kappa_{l}}\right)\left\{\tilde{f}_{0} R_{1}(k, \rho)+\sum_{n=1}^{\infty}\left[\left(\tilde{f}_{n}(k) R_{n+1}(k, \rho)+\tilde{\alpha}_{n}(k)\left(\frac{R_{n}(k, \rho)}{\kappa_{l} \rho}-\frac{1}{n} R_{n-1}(k, \rho)\right)\right) \cos (n \phi)\right.\right. \\
\left.\left.+\left(\tilde{e}_{n}(k) R_{n+1}(k, \rho)+\tilde{\beta}_{n}(k)\left(\frac{R_{n}(k, \rho)}{\kappa_{l} \rho}-\frac{1}{n} R_{n-1}(k, \rho)\right)\right) \sin (n \phi)\right]\right\}, \\
E_{z}(\mathbf{r})=\int_{-\infty}^{\infty} \frac{d k}{\sqrt{2 \pi}} e^{\mathrm{i} k z}\left\{\tilde{e}_{0}(k) R_{0}(k, \rho)+\sum_{n=1}^{\infty}\left[\tilde{e}_{n}(k) R_{n}(k, \rho) \cos (n \phi)+\tilde{f}_{n}(k) R_{n}(k, \rho) \sin (n \phi)\right]\right\},
\end{gathered}
$$

where $R_{n}$ is either a regular or modified Bessel function,

$$
R_{n}(k, \rho)= \begin{cases}J_{n}\left(\kappa_{l}(k) \rho\right) & \text { if } \operatorname{sgn}\left(k^{2}-k_{l}^{2}\right)<0, \\ I_{n}\left(\kappa_{l}(k) \rho\right) & \text { otherwise }\end{cases}
$$

where $k_{l}$ is the wave number for the given mode in the cavity (in this case $k_{l} \equiv \omega_{l} / c \approx 8.34 \mathrm{~m}^{-1}$ ), and $n$ is the order of the Bessel function. The function $\kappa_{l}$ is dependent on $k_{l}$ and $k$,

$$
\kappa_{l}(k)^{2}=\left|k^{2}-k_{l}^{2}\right|
$$

\section{APPENDIX B: COMPUTATION OF THE TAYLOR MAPS}

The Hamiltonian takes the following form:

$$
\begin{aligned}
H= & -\sqrt{\left(\frac{1}{\beta_{0}}+\delta\right)^{2}-\left(p_{x}-a_{x}\right)^{2}-\left(p_{y}-a_{y}\right)^{2}-\frac{1}{\gamma_{0}^{2} \beta_{0}^{2}}} \\
& -a_{s}+\frac{\delta}{\beta_{0}}+p_{s}
\end{aligned}
$$

where $a_{x, y, s}(x, y, z, s)$ are the normalized vector potentials, $x, p_{x}, y, p_{y}$ are the canonical variables describing transverse positions and conjugate momenta, and $z$ and $\delta$ are the relative position to the synchronous particle and relative energy defined by

$$
\begin{aligned}
& \delta=\frac{E}{p_{0} c}-\frac{1}{\beta_{0}}, \\
& z=\frac{s}{\beta_{0}}-c t .
\end{aligned}
$$

$E, p_{0}, \beta_{0}, s$, and $t$ define the particle energy, reference momentum, reference speed as a fraction of $c$, position on reference path and time, respectively. $\gamma_{0}$ and $\beta_{0}$ are the reference relativistic factors corresponding to the reference momentum. The Hamiltonian is extended to four conjugate pairs, with the fourth $\left\{s, p_{s}\right\}$ allowing motion along the reference trajectory through an $s$-dependent vector field $[19,26]$.

Production of trajectories through the fields, with varying initial variables, allows for a greater understanding of the fundamental dynamics of the cavity. A numerical implementation WFR integration [19] has previously been presented for this cavity in [27], and it was shown that multiple kicks throughout the cavity lead to small zeroth order displacement terms in the transfer map.

A Taylor map expresses the relationship between the initial and final state variables in the form of a Taylor series,

$$
\left(\begin{array}{r}
x_{1}^{f} \\
x_{2}^{f} \\
x_{3}^{f} \\
x_{4}^{f} \\
x_{5}^{f} \\
x_{6}^{f}
\end{array}\right)=\left(\begin{array}{l}
f_{1}\left(x_{1}^{i}, \ldots, x_{6}^{i}\right) \\
f_{2}\left(x_{1}^{i}, \ldots, x_{6}^{i}\right) \\
f_{3}\left(x_{1}^{i}, \ldots, x_{6}^{i}\right) \\
f_{4}\left(x_{1}^{i}, \ldots, x_{6}^{i}\right) \\
f_{5}\left(x_{1}^{i}, \ldots, x_{6}^{i}\right) \\
f_{6}\left(x_{1}^{i}, \ldots, x_{6}^{i}\right)
\end{array}\right),
$$

where

$$
f_{k}\left(x_{1}^{i}, \ldots, x_{6}^{i}\right)=\sum_{i_{1}, \ldots, i_{6}=0}^{\sum_{j}^{6} i_{j}=\text { Order }} A_{k, i_{1}, \ldots, i_{6}} \prod_{j=1}^{6}\left(x_{j}^{i}\right)^{i_{j}},
$$


where the order of a Taylor map is determined by the largest total power of any term in the series. $x_{j}^{i}$ are the initial values of the canonical variables, $i_{j}$ are the exponents, and $A_{k, i_{1} \ldots i_{6}}$ is the coefficient of term defined by the exponents in the series $f_{k}$.

\section{APPENDIX C: SYMPLECTIC ERROR}

Truncating the Taylor map introduces a symplectic error, which leads to a failure to conserve the phase space volume during tracking. The truncation of the map, to a given order, leads to a varying numerical precision dependent upon the amplitude of the initial variables. The Jacobian of a map is defined by

$$
J\left(\vec{x}^{i}\right)=\left(\begin{array}{ccc}
\frac{\partial x_{1}^{f}}{\partial x_{1}^{i}} & \cdots & \frac{\partial x_{1}^{f}}{\partial x_{6}^{i}} \\
\vdots & \ddots & \vdots \\
\frac{\partial x_{6}^{f}}{\partial x_{1}^{i}} & \cdots & \frac{\partial x_{6}^{f}}{\partial x_{6}^{i}}
\end{array}\right),
$$

where $\vec{x}^{i}$ and $\vec{x}^{f}$ are the initial and final values of the canonical variables. The Jacobian is said to be symplectic if the following condition holds:

$$
J^{T}\left(\vec{x}^{i}\right) \cdot S \cdot J\left(\vec{x}^{i}\right)=S,
$$

where $J^{T}$ is the transpose of the Jacobian and $S$ is defined by

$$
S=\left(\begin{array}{cccccc}
0 & 1 & 0 & 0 & 0 & 0 \\
-1 & 0 & 0 & 0 & 0 & 0 \\
0 & 0 & 0 & 1 & 0 & 0 \\
0 & 0 & -1 & 0 & 0 & 0 \\
0 & 0 & 0 & 0 & 0 & 1 \\
0 & 0 & 0 & 0 & -1 & 0
\end{array}\right) .
$$

The symplectic error is given by the coefficients of the matrix $E$ defined as

$$
E\left(\vec{x}^{i}\right)=J^{T}\left(\vec{x}^{i}\right) \cdot S \cdot J\left(\vec{x}^{i}\right)-S .
$$

From a Taylor map produced with a step size of $0.01 \mathrm{~m}$ and truncation order eight, the symplectic error up to eighth order is shown in Fig. 8, evaluated at a single point in phase space. A fuller study would be required in order to conclude on the complete phase space encountered within the machine. The error values decrease from second to eighth order. The first order map has the least symplectic error as its only source of error comes from the machine precision (double precision) rather than the series truncation, hence the symplecticity is at the level of $10^{-15}$.

Considering the matrix $E\left(\vec{x}^{i}\right)$ as a function of the initial conditions it can be evaluated to find the maximum over the

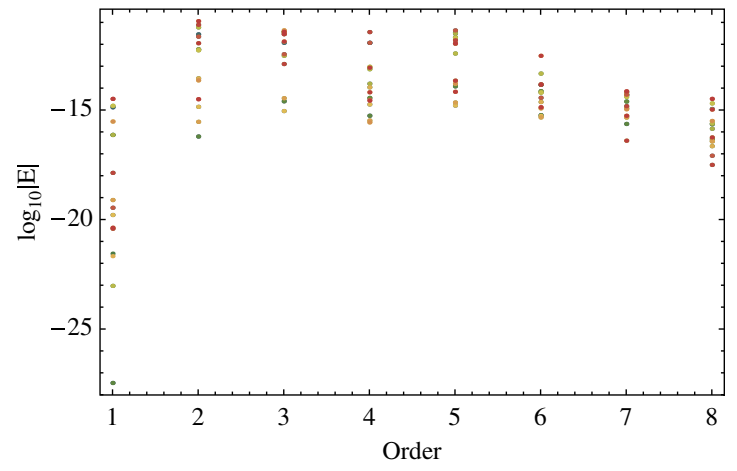

FIG. 8. Symplectic error, $E$, absolute matrix terms for initial values $(x, y, z)=0.01$ and $\left(p_{x}, p_{y}, \delta\right)=0.0001$ from eighth order in Taylor series map. Voltage normalized to $4.155 \mathrm{MV}$.

hypersphere of the 6D phase space. Such an evaluation removes the limitations brought about by considering one point in phase space. Evaluating $E$ over the phase space ranges, as determined by the $6 \mathrm{D}$ tracking at large amplitude, given by $(x, y)=[-0.02,0.02] \mathrm{m}$, $\left(p_{x}, p_{y}\right)=[-0.0001,0.0001], z=[-0.1,0.1] \mathrm{m}$ and $\delta=$ $[-0.0001,0.0001]$, and determining the maximum determines a position independent measure of the symplectic error. For the eighth order 4.155 MV map this is given by $6.05 \times 10^{-13}$. The fact that this value is greater than double precision would suggest that the symplectic error would have a visible effect in the numerical tracking at a faster rate than with symplectic numerical tracking. However, such an evaluation does not equate to a quantifiable effect in terms of error in tracking; therefore, it is impossible to judge the impact of the symplectic error without experimentally testing the Taylor map in long-term tracking. If a Taylor map's symplectic error were small, it would be expected that it would give a good agreement for some finite number of turns with a symplectic model that contains the same dynamics.

[1] L. Rossi and R. De Maria, in Proceedings of the 2nd International Particle Accelerator Conference, San Sabastian, Spain, 2011.

[2] K. Oide and K. Yokoya, Phys. Rev. A 40, 315 (1989).

[3] Y.-P. Sun, R. Assmann, J. Barranco Garcia, R. Tomás Garcia, T. Weiler, F. Zimmermann, R. Calaga, and A. Morita, Phys. Rev. ST Accel. Beams 12, 101002 (2009).

[4] B. Hall, P. K. Ambattu, G. Burt et al., in Proceedings of the 3rd International Particle Accelerator Conference, New Orleans, LA, 2012 (IEEE, Piscataway, NJ, 2012).

[5] R. Calaga, S. A. Belomestnykh, I. Ben-Zvi, J. Skaritka, Q. Wu and B.P. Xiao, in Proceedings of the 4th International Particle Accelerator Conference, Shanghai, China, 2013.

[6] D. A. Goldberg and G. R. Lambertson, AIP Conf. Proc. 249, 537 (1992). 
[7] J. Barranco Garcia, R. Calaga, R. De Maria et al., in Proceedings of the 3rd International Particle Accelerator Conference, New Orleans, LA (IEEE, Piscataway, 2012).

[8] K. Makino and M. Berz, Nucl. Instrum. Methods Phys. Res., Sect. A 558, 346 (2006).

[9] R. Kleiss, F. Schmidt, and F. Zimmermann, Part. Accel. 41, 117 (1993).

[10] M. Berz, Nucl. Instrum. Methods Phys. Res., Sect. A 363, 100 (1995).

[11] D. Abell, Phys. Rev. ST Accel. Beams 9, 052001 (2006).

[12] J. Irwin, AIP Conf. Proc. 326, 662 (1995).

[13] D. Abell., Ph.D. thesis, College Park, University of Maryland, MD, 1995.

[14] E. Forest, J. Phys. A 39, 5321 (2006).

[15] ANSYS, "HFSS," http://www.ansys.com/ (2011).

[16] A. J. Dragt, "Lie methods for nonlinear dynamics with applications to accelerator physics," www.physics.umd .edu/dsat (2011).

[17] M. Venturini and A. Dragt, Nucl. Instrum. Methods Phys. Res., Sect. A 427, 387 (1999).
[18] C. E. Mitchell and A. J. Dragt, Phys. Rev. ST Accel. Beams 13, 064001 (2010)

[19] Y. K. Wu, E. Forest, and D. S. Robin, Phys. Rev. E 68, 046502 (2003).

[20] H. Yoshida, Phys. Lett. A 150, 262 (1990).

[21] W. K. H. Panofsky and W. A. Wenzel, Rev. Sci. Instrum. 27, 967 (1956).

[22] J. Barranco Garcia, R. De Maria, A. Grudiev, R. Tomás Garcia, R. B. Appleby, and D. R. Brett (to be published).

[23] H. Grote, F. Schmidt, and L. H. A. Leunissen, CERN, Tech. Rep. No. LHC-Project-Note-197, 1999.

[24] E. Mcintosh and R. De Maria, "The SixDesk run environment for SixTrack," sixtrack-ng.web.cern.ch/ sixtrack-ng/ (2012).

[25] S. Fartoukh and R. De Maria, in Proceedings of the 3rd International Particle Accelerator Conference, New Orleans, LA (IEEE, Piscataway, NJ, 2012).

[26] E. Forest, Beam Dynamics: A New Attitude and Framework (CRC Press, Boca Raton, 1998).

[27] D. R. Brett, R. B. Appleby, G. Burt, and B. Hall, Nucl. Instrum. Methods Phys. Res., Sect. A 734, 79 (2014). 